\title{
Evidence of potent antibacterial effect of fermented papaya leaf against opportunistic skin pathogenic microbes
}

\author{
Mohd Danial, A., Koh, S.P., Abdullah, R. and Azali, A. \\ Food Science and Technology Research Centre, MARDI Headquarter, 43400 Serdang, Selangor, Malaysia.
}

\section{Article history:}

Received: 11 April 2020

Received in revised form: 22

July 2020

Accepted: 28 September 2020

Available Online: 27

December 2020

Keywords:

Bacterial pathogens,

Efficacy,

Fermented papaya leaf,

Minimum inhibitory

concentration,

Supernatant

DOI:

https://doi.org/10.26656/fr.2017.4(S6).011

\begin{abstract}
The papaya leaf juice has been long practised as a traditional remedy to cure ailments due to its medicinal properties. The objective of this research is to study the effectiveness of fermented papaya leaf to inhibit the growth of pathogenic bacteria and yeast: Staphylococcus aureus, Pseudomonas aeruginosa, Propionibacterium acnes and Candida albicans. The efficacy of fermented papaya leaf against selected pathogenic microbes was evaluated using agar well diffusion assay, broth microdilution assay and time-kill test. Evidence from data collected confirmed that fermented papaya leaf supernatant showed more pronounced antibacterial and antifungal effect than papaya leaf alone. Generally, fermented papaya leaf supernatant demonstrated potent antimicrobial effect against all bacterial pathogens tested particularly $P$. aeruginosa followed by $P$. acne and $S$. aureus. However, it was found that fermented papaya leaf was less effective against Candida albicans. It needs 4- to 7-folds higher concentration to inhibit 50\% C. albicans growth than the bacteria. The antibacterial compounds produced in the supernatant appeared to have some bactericidal effect against $P$. aeruginosa, $P$. acne and $S$. aureus with the minimum inhibitory concentration $\left(\mathrm{MIC}_{>99}\right)$ of $16 \%, 50 \%$ and $60 \%$, respectively. Particularly, the fermented papaya leaf supernatant at $60 \%$ concentration showed $100 \%$ inhibition rate within 30 mins against $P$. aeruginosa. However, it needs a longer time to show the same inhibition effect against $S$. aureus and $P$. acne, which was about 2-6 h. The potent killing effect of fermented papaya leaf showed a potential use in skincare application to control pathogenic microbe infection
\end{abstract}

\section{Introduction}

Papaya (Carica papaya Linn) is cultivated mainly in all tropical and sub-tropical countries around the world. Various parts of papaya such as the leaves, seeds, flesh, latex and skin were known to have many benefits to human being and have been long used as a traditional remedy to cure ailments. Many studies have been conducted to look at the medical properties of papaya leaf not only on diseases related to human but also to animals. The papaya leaf was found to have antidiabetic (Juárez-Rojop et al., 2014), antimicrobial (Baskaran et al., 2012; Jafari et al., 2016), anti-inflammatory (Gupta et al., 2017) and anti-tumour (Otsuki et al., 2010) and antioxidant activities (Okoko and Ere, 2012). Both aqueous and solvent papaya leaf extract was reported to contain numerous bioactive compounds such as alkaloids, steroids, quinones, tannins (Juárez-Rojop et al., 2014), prunasin, sambunigrin (Seigler et al., 2002) and flavonoids (Vuong et al., 2013). Nevertheless, papaya leaf juice is very bitter and hard to be consumed even though many people knew its various pharmacological properties.

Skin infection caused by either bacteria or yeast is a worldwide problem to human and animal. All age group can be infected, either normal or immunocompromised patients. Some of the common bacteria and yeast isolated were from Staphylococcus, Pseudomonas, Propionibacterium and Candida genera (Cogen et al., 2008). At present patients are treated with antibiotics. However, some of the bacterial and yeast are getting resistant to the antibiotics (Cogen et al., 2008). In addition, patients with drug therapy are generally exposed to the risk of drug-drug interaction which may reduce the efficacy of the drug or affect the health of the patients (Gupta et al., 2013). This is a serious problem as antibiotics are used clinically either for oral or topical treatment for both bacterial and yeast infection. Therefore, the use of natural antimicrobial compounds 
which are generally safe would be beneficial.

Fermentation is a degradation of nutritional compounds in raw commodities through the action of a multiple hydrolytic enzymes secreted by the microorganisms. Depending on the strains, numerous primary and secondary metabolites with various functional properties and that contribute to the flavour were produced by selective microorganisms used during the fermentation process. Thus, many food industries use single or mixtures of pure cultures under controlled fermentation condition for the production of a fermented product with the desired characteristics which are safe for the consumer. Some of the pure cultures used for the preparation of known fermented foods include nontoxigenic Aspergillus oryzae strains for preparation of soy sauce (Hoang et al., 2016), Rhizopus for tempeh (Hartanti et al., 2015), Lactobacillus for yoghurt (Ertem and Cakmakci, 2018) and Saccharomyces for wine (Marsit and Dequin, 2015). In addition to this, many studies are now applying various new raw materials as a substrate to produce a novel fermented product with better health properties. Therefore, the objective of this research is to study the effectiveness of fermented papaya leaf to inhibit the growth of opportunistic bacterial and yeast pathogens: Staphylococcus aureus, Pseudomonas aeruginosa, Propionibacterium acnes and Candida albicans.

\section{Materials and methods}

\subsection{Preparation of non-fermented and fermented papaya leaf}

Non-fermented and fermented papaya leaf was prepared as described by Koh et al. (2017). After fermentation, papaya leaf was subjected to filtration and centrifugation at $10,000 \mathrm{rpm}, 4^{\circ} \mathrm{C}$ for 10 mins. The supernatant was collected and filtered sterilized using sterile $0.22 \mu \mathrm{m}$ cellulose acetate syringe filter and kept at $-80^{\circ} \mathrm{C}$ until used.

\subsection{Preparation of bacterial and yeast cell suspension}

Staphylococcus aureus (ATCC 49775'1M), Propionibacterium acnes (ATCC 6919 ${ }^{\mathrm{TM}}$ ) and Candida albicans (ATCC 26310 ${ }^{\mathrm{TM}}$ ) were purchased from American Type Culture Collection (ATCC), whereas, Pseudomonas aeruginosa was isolated from mastitis cow. The glycerol stock of $S$. aureus and $P$. aeruginosa was inoculated in Mueller Hinton (MH) broth and $C$. albicans was inoculated in potato dextrose (PD) broth, followed by incubation at $30^{\circ} \mathrm{C}$ with the agitation rate of $200 \mathrm{rpm}$ for $18 \mathrm{hrs}$. P. acne was grown in a mixture of tryptic soy (TS) and Lab-Lemco broth in an anaerobic condition at $35^{\circ} \mathrm{C}$ for four days. The cells were harvested by centrifugation and washed with sterile $0.85 \%$ sodium chloride $(\mathrm{NaCl})$. Finally, the density of the cell suspension was adjusted with sterile $0.85 \% \mathrm{NaCl}$ to $10^{8}$ $\mathrm{CFU} / \mathrm{mL}$.

\subsection{Agar well diffusion assay}

A bacterial and yeast cell suspension was spread uniformly on the solid agar medium using cotton swab and left dried at room temperature. The wells were cut using sterile Pasteur pipet and soft agar was loaded to seal the bottom edge of the well. An amount of $50 \mu \mathrm{L}$ of the fermented papaya leaf supernatant (FPLS) was loaded and kept in chilled condition for two hours to allow diffusion of the sample into the agar. Then another $50 \mu \mathrm{L}$ FPLS was added into well. The agar plate was then incubated at $37^{\circ} \mathrm{C}$ for $24 \mathrm{hrs}$ for $S$. aureus, $P$. aeruginosa and $C$. albicans, while $P$. acnes was incubated under anaerobic conditions for a duration of 4 days. A clear zone diameter around the well which indicated the microbial inhibition was measured at two perpendicular directions. Penicillin-Streptomycin and acetic acid were used as a positive control. All experiments were done with three replicates

\subsection{Determination of minimum inhibitory concentration at level of $M I C_{50}$ and $M I C_{>99}$}

A mixture of different concentration of FPLS (0$100 \%$ ) and growth medium broth was loaded into sterile microtiter plate (total volume $90 \mu \mathrm{l} /$ well). Later, $10 \mu \mathrm{L}$ of cell suspension was added (final CFU $10^{6} / \mathrm{mL}$ ) and the plate was sealed with paraffin film to avoid spillage. The culture was incubated as mentioned above and shaking at $50 \mathrm{rpm}$. After incubation, a serial dilution was performed in sterile $0.85 \% \mathrm{NaCl}$ and plated on solid agar medium followed by incubation for $24 \mathrm{hrs}$ to determine the number of viable cells after the treatment. The medium with no viable cell after $24 \mathrm{hrs}$ were further incubated and observed up to $48 \mathrm{hrs}$. The concentration of FPLS inhibited $50 \%$ of microorganism growth $\left(\mathrm{MIC}_{50}\right)$ was calculated using GraphPad Prism 5 software. The $\mathrm{MIC}_{>99}$ was determined based on the lowest concentration that totally killed the microorganisms. All experiments were done with three replicates.

\subsection{Time-kill study}

A tube containing a mixture of sterile growth medium broth and FPLS (final concentration 60\%) was inoculated with the bacterial cell suspension. The final concentration of cell suspension was approximately $10^{6}$ $\mathrm{CFUs} / \mathrm{mL}$. The broth without FPLS was used as a control. The mixture was incubated at $37^{\circ} \mathrm{C}$ for $10 \mathrm{hrs}$ and at certain time interval, a total of $100 \mu \mathrm{L}$ sample was removed for $\mathrm{CFU}$ analysis. A serial dilution was 
conducted before the cell was grown on agar medium for 24-48 hrs after which the visible colonies were counted. All experiments were done with three replicates.

\subsection{Statistical analysis}

The data were analyzed using one-way analysis of variance (ANOVA) and t-test to compare the means for each treatment. $\mathrm{p}<0.05$ was accepted as a significant difference. The GraphPad Prism 5 (GraphPad Software Inc. California, USA) was used in the analyses.

\section{Results and discussion}

\subsection{Agar well diffusion assay}

Agar well diffusion assay was used as a preliminary assay to examine the potential of non-fermented and fermented papaya leaf for growth inhibition of $S$. aureus, $P$. aeruginosa, $P$. acne and $C$. albicans. It was noted that none of these microbes were affected by the nonfermented papaya leaf (Table 1). However, fermented papaya leaf showed inhibition activity toward these selected pathogenic microorganisms, particularly against $S$. aureus and $P$. aeruginosa but no inhibition was observed against $P$. acne and $C$. albicans using this assay. There was no significant different $(p>0.05)$ in inhibition zone diameter between $S$. aureus $(1.50 \mathrm{~cm}$ inhibition zone) and $P$. aeruginosa $(1.40 \mathrm{~cm}$ inhibition zone) on agar plate treated with fermented papaya leaf supernatant. Indeed, the inhibition activity against $P$. aeruginosa was not significantly different when compared to Penicillin-Streptomycin (1\%) and 2\% acetic acid (control). However, the inhibition zone of $S$. aureus was significantly smaller $(\mathrm{p}<0.05)$ than PenicillinStreptomycin (1\%) which about $28 \%$. Previous studies had reported that the antimicrobial activity of papaya leaf was varied depending on the extraction method (Khan et al., 2012) and the bacterial strains (Cho and Maung, 2017). The antibacterial activity was high when the extraction was carried out using ethanol but has no effect with water against gram-positive and negative strains (Awah et al., 2017). In this study, the negative antimicrobial effect of the non-fermented papaya leaf could be due to the application of the water extraction

Table 1. Antimicrobial activity of fermented papaya leaf supernatant against opportunistic bacterial and yeast pathogen using agar well diffusion assay.

\begin{tabular}{lcccc}
\hline \multirow{2}{*}{ Antimicrobial agent } & \multicolumn{4}{c}{ Inhibition zone diameter $(\mathrm{cm})$} \\
\cline { 2 - 5 } & S. aureus & P. aeruginosa & P. acne & C. albicans \\
\hline Penicillin- Streptomycin (1\%) & $2.10 \pm 0.12^{*}$ & $1.95 \pm 0.42^{*}$ & $1.70 \pm 0.36$ & NI \\
Acetic acid (2\%) & $1.80 \pm 0.22$ & $1.60 \pm 0.21^{*}$ & $1.10 \pm 0.16$ & NI \\
Papaya Leaf: & & & & \\
Non-fermented & NI & NI & NI & NI \\
Fermented & $1.50 \pm 0.15 \dagger$ & $1.40 \pm 0.10^{*} \dagger$ & NI & NI \\
\hline
\end{tabular}

Data are means of triplicate \pm standard deviation. $(*)$ indicate significant different $(p<0.05)$ within treatments and $(f)$ between pathogenic microorganisms. NI: no inhibition 
concentration to give the same effects on C. albicans. Nevertheless, FPLS cannot kill C. albicans totally with no $\mathrm{MIC}_{>99}$ value observed. On the other hand, the antibacterial compounds produced in the FPLS appeared to have some bactericidal effect against $P$. aeruginosa, $P$. acne and $S$. aureus with the minimum inhibitory concentration $\left(\mathrm{MIC}_{>99}\right)$ of $16 \%, 50 \%$ and $60 \%$,
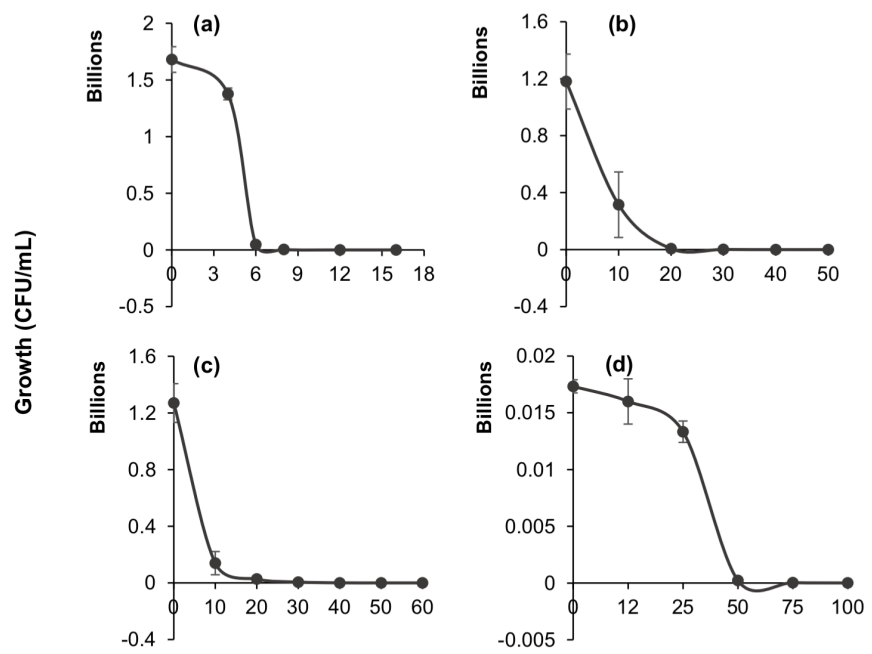

Concentration (\%)

Figure 1. Effect of different concentration of fermented papaya leaf supernatant on the growth inhibition pattern of (a) P. aeruginosa (b) P. acne, (c) S. aureus and (d) C. albicans

Table 2. $\mathrm{MIC}_{50}$ and $\mathrm{MIC}_{>99}$ of fermented papaya leaf supernatant for controlling the growth of $P$. aeruginosa, $P$. acne, S. aureus and C. albicans

\begin{tabular}{lcc}
\hline Bacterial and yeast strain & $\mathrm{MIC}_{50}(\%)$ & $\mathrm{MIC}_{>99}(\%)$ \\
\hline P. aeruginosa & 4.5 & 16 \\
P. acne & 8.5 & 50 \\
S. aureus & 4.2 & 60 \\
C. albicans & 28.4 & - \\
\hline
\end{tabular}

respectively.

\subsection{Time-kill study}

Time-kill pattern of fermented papaya leaf supernatant (FPLS) against the three bacteria pathogens is shown in Figure 2. The highest $\mathrm{MIC}_{>99}$ value of FPLS ( $60 \%$ concentration) was selected to compare the time needed to kill each of the bacteria completely. It was noticed that there was a $1.9 \log$ growth reduction when $P$. aeruginosa cell was exposed to the FPLS. Indeed, the killing time was very short to eliminate $P$. aeruginosa, which was about 30 mins. In contrast, FPLS need about $2 \mathrm{hrs}$ to cause $100 \%$ inhibition against $P$. acne. The different phenomenon was observed for $S$. aureus during the time-kill study. There was a gradual growth reduction of $S$. aureus during $8 \mathrm{hrs}$ exposure to the FPLS. The growth was reduced from $7.3 \log$ to $2.2 \log$ at the initial $4 \mathrm{hrs}$ and finally, no viable cell was found after 6 hrs exposure. The ability of the FPLS to kill the bacteria instantly is important. This is particularly to reduce the possibility of the bacteria to develop resistance to the antimicrobial agents. It was reported that prolong exposure of dermatophyte T. rubrum to the sub-inhibitory concentration of antifungal drug has reduced the susceptibility to the antifungal drug (Ghelardi et al., 2014).

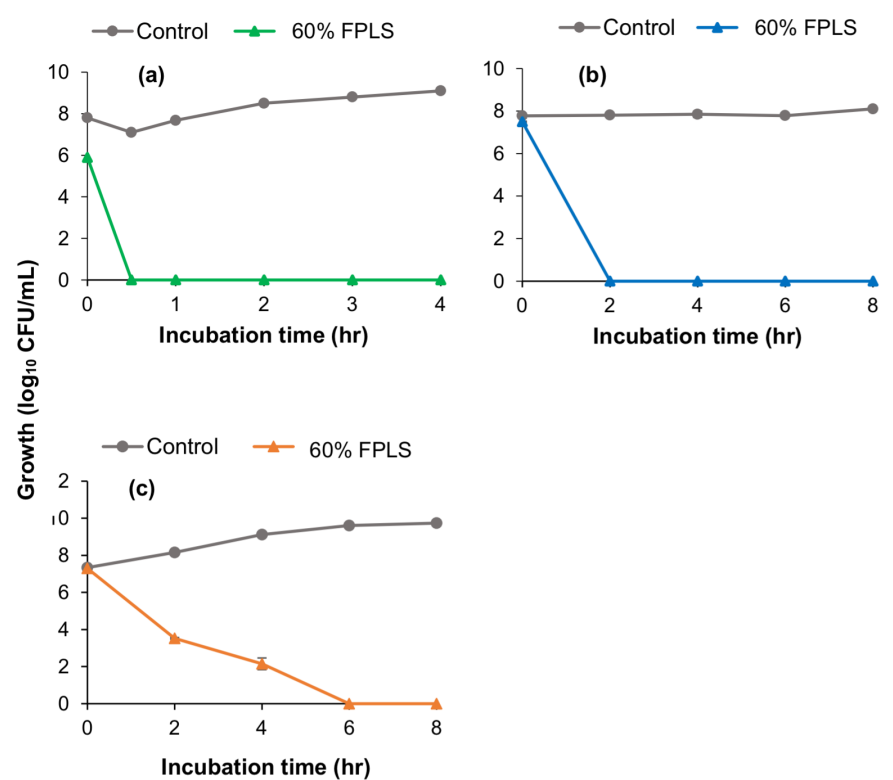

Figure 2. Time-kill pattern of fermented papaya leaf (FPLS) supernatant at $60 \%$ concentration against (a) P. aeruginosa, (b) P. acne and (c) S. aureus.

\section{Conclusion}

Fermented papaya leaf was very effective against $P$. aeruginosa growth, followed by $P$. acne and $S$. aureus. The potent killing effect of fermented papaya leaf supernatant showed a potential use as bioactive ingredient in skincare application to control pathogenic microbe infection. Future study will focus on the identification of bioactive metabolites present in fermented papaya leaf supernatant with antimicrobial properties.

\section{Acknowledgement}

The authors would like to acknowledge the financial support provided by Ministry of Agriculture and AgroBased Industry under Malaysia Government Development Fund RMK-11 (P21003004050001).

\section{References}

Awah, N.S., Agu, K.C., Ilkedinma, J.C., Uzoechi, A.N., Eneite, H.C., Victor-Aduloju, A.T., Umeoduagu, N.D., Onwuatuegwu, J.T.C. and Ilikannu, S.O. (2017). Antibacterial activities of the aqueous and ethanolic extracts of the male and female Carica papaya leaves on some pathogenic bacteria. Bioengineering and Bioscience, 5(2), 25-29. 
Baskaran, C., Ratha bai, V., Velu, S. and Kumaran, K. (2012). The efficacy of Carica papaya leaf extract on some bacterial and a fungal strain by well diffusion method. Asian Pacific of Tropical Disease, 2(2), S658-662. https://doi.org/10.1016/S2222-1808 (12)60239-4

Cho, H.N. and Maung, N.N. (2017). Study on the antimicrobial and antioxidant activities of Carica papaya Linn (Thin Baw). International Journal of Scientific Engineering and Scientific Research, 6(4), 610-613.

Cogen, A.L., Nizet, V. and Gallo, R.L. (2008). Skin microbiota: a source of disease or defence? British Journal of Dermatology, 158(3), 442-455. https:// doi.org/10.1111/j.1365-2133.2008.08437.x

Ertem, H. and Cakmakci, S. (2018). Shelf life and quality of probiotic yogurt produced with Lactobacillus acidophillus and Gobdin. International Journal of Food Science and Technology, 53(3), 776 -783. https://doi.org/10.1111/ijfs. 13653

Ghelardi, E., Celandroni, F., Gueye, A., Salvetti, S., Senesi, S., Bulgheroni, A. and Mailland, F. (2014). Potential of ergosterol synthesis inhibitors to cause resistance or cross-resistance in Trichophyton rubrum. Antimicrobial Agents and Chemotherapy, 58(5), 2825-2829. https://doi.org/10.1128/ AAC.02382-13

Gupta, A., Patil, S.S. and Pendharkar, N. (2017). Antimicrobial and anti-inflammatory activity of aquoes extract of Carica papaya. Journal of Herbmed Pharmacology, 6(4), 148-152.

Gupta, A.K., Paquet, M. and Simpson, F.C. (2013). Therapies for the treatment of onychomycosis. Clinics in Dermatology, 31(5), 544-554. https:// doi.org/10.1016/j.clindermatol.2013.06.011

Hartanti, A.T., Rahayu, G. and Hidayat, I. (2015). Rhizopus species from fresh tempeh collected from several regions in Indonesia. HAYATI Journal of Biosciences, 22, 136-142. https://doi.org/10.1016/ j.hjb.2015.10.004

Hoang, N.X., Ferng, S., Ting, C-H., Huang, W-H., Chiou, R.Y-Y. and Hsu, C-H. (2016). Optimizing the initial moromi fermentation conditions to improve the quality of soy sauce. LWT-Food Science and Technology, 74, 242-250. https://doi.org/10.1016/ j.lwt.2016.07.049

Jafari S., Meng, G.Y., Rajion, M.A., Jahromi, M.F. and Ebrahimi, M. (2016). Manipulation of rumen microbial fermentation by polyphenol rich solvent reactions from papaya leaf to reduced green-house gas methane and biohydrogenation of C18 PUFA. Journal of Agricultural and Food Chemistry, 64(22),
4522-4530. https://doi.org/10.1021/acs.jafc.6b00846

Juárez-Rojop, I.E., Tovilla-Zárate, C.A., AguilarDominguez, D.E., Roa-de la Fuente, L.F., LobatoGarcia, C.E., Ble-Castillo, J.L., Lopez-Meraz, L., Diaz-Zagoya, J.C. and Bermudez-Ocana, D.Y. (2014). Phytochemical screening and hypoglycemic activity of Carica papaya leaf in streptozotocininduced diabetic rats. Brazilian Journal of Pharmacognosy, 24, 341-347. https:// doi.org/10.1016/j.bjp.2014.07.012

Khan, J.A., Yadav, J., Srivastava, Y. and Pal, P.K. (2012). In vitro evaluation of antimicrobial properties of Carica papaya. International Journal of Biology, Pharmacy and Allied Sciences, 1(7), 933945.

Koh, S.P., Aziz, N., Sharifudin, S.A, Abdullah, R., Hamid, N.S.A. and Sarip, J. (2017). Potential of fermented papaya beverage in the prevention of foodborne illness incidence. Food Research, 1(4), 109-113. https://doi.org/10.26656/fr.2017.4.022

Marsit, S. and Dequin, S. (2015). Diversity and adaptive evolution of Saccharomyces wine yeast: a review. FEMS Yeast Research,15(7), 1-12. https:// doi.org/10.1093/femsyr/fov067

Okoko, T. and Ere, D. (2012). Reduction of hydrogen peroxide-induced erythrocyte damage by Carica papaya leaf extract. Asian Pacific Journal of Tropical Biomedicine, 2(6), 449-453. https:// doi.org/10.1016/S2221-1691(12)60074-4

Otsuki, N., Dang, N.H., Kumagai, E., Kondo, A., Iwata, S. and Morimoto, C. (2010). Aqueous extract of Carica papaya leaves exhibits anti-tumor activity and immunomodulatory effects. Journal of Ethnopharmacology, 127(3), 760-767. https:// doi.org/10.1016/j.jep.2009.11.024

Parvez, Md. A.K., Saha, K., Rahman, J., Ara Munmun, R., Rahman, Md. A., Dey, S.K., Rahman, Md. S., Islam, S. and Shariare, M.H. (2019). Antibacterial activities of green tea crude extracts and synergistic effects of epigallocatechingallate (EGCG) with gentamicin against MDR pathogens. Heliyon, 5(7), e02126.

https://doi.org/10.1016/ j.heliyon.2019.e02126

Pejin, J., Radosavljević, M., Kocić-Tanackov, S., Djukić -Vuković, A. and Mojović, L. (2017). Lactic acid fermentation of brewer's spent grain hydrolysate by Lactobacillus rhamnosus with yeast extract addition and $\mathrm{pH}$ control. Journal of the Institute of Brewing, 123(1), 98-104. https://doi.org/10.1002/jib.403

Rashad, Y.M., Al-Askar, A.A., Ghoneem, K.M., Saber, W.I.A. and Hafez, E.E. (2017). Chitinolytic Streptomyces griseorubens E44G enhances the 
biocontrol efficacy against Fusarium wilt disease of tomato. Phytoparasitica, 45(2), 227-237. https:// doi.org/10.1007/s12600-017-0580-3

Seigler, D.S., Pauli, G.F., Nahrstedt, A. and Leen, R. (2002). Cyanogenic allosides and glucosides from Passiflora edulis and Carica papaya. Phytochemistry, 60(8), 873-882. https:// doi.org/10.1016/S0031-9422(02)00170-X

Sheih, I.C., Fang, T.J., Wu, T.K. and Chen, R.Y. (2014). Effects of fermentation on antioxidant properties and phytochemical composition of soy germ. Journal of the Science of Food and Agriculture, 94(15), 31633170. https://doi.org/10.1002/jsfa.6666

Souza, M.D., Dimitrov, S., Ivanova, I., Dora, B., Melo, G.D., Chobert, J. and Haertl, T. (2015). Improving safety of salami by application of bacteriocins produced by an autochthonous Lactobacillus curvatus isolate. Food Microbiology, 46, 254-262. https://doi.org/10.1016/j.fm.2014.08.004

Velićanski, A.S., Cvetković, D.D., Markov, S.L., Tumbas, V.T. and Vulić, J.J. (2014). Antioxidant and antibacterial activity of the beverage obtained by fermentation of sweetened lemon balm (Melissa officinalis L.) tea with symbiotic consortium of bacteria and yeasts. Food Technology and Biotechnology, 52(4), 420-429. https:// doi.org/10.17113/ftb.52.04.14.3611

Vuong, V.V., Hirun, S., Roach, P.D., Bowyer, M.C., Philips, P.A. and Scarlett, C.J. (2013). Effect of extraction conditions on total phenolic compounds and antioxidant activities of Carica papaya leaf aqueous extracts. Journal of Herbal Medicine, 3(3), 104-111. https://doi.org/10.1016/ j.hermed.2013.04.004 